\title{
Análise do Desenvolvimento Morfológico da Blenda Polimérica PBT/ABS durante as Etapas de Mistura por Extrusão e Moldagem por Injeção
}

\author{
Edson N. Ito, Luiz A. Pessan, Elias Hage Jr. \\ Departamento de Engenharia de Materiais, UFSCar
}

José A. Covas

Depto de Engenharia de Polímeros, Uminho, Portugal

\begin{abstract}
Resumo: O enfoque principal deste trabalho foi observar o desenvolvimento da morfologia de fases da blenda binária PBT/ABS e desta blenda compatibilizada pela adição de um copolímero acrílico reativo, durante a etapa de mistura por extrusão e de moldagem por injeção. A evolução da morfologia das blendas, durante a etapa de mistura, foi analisada através do uso de amostras coletadas de uma extrusora de rosca dupla co-rotacional, com acessório especialmente projetado para coleta in line. A morfologia observada nas amostras obtidas por injeção foi realizada utilizando amostras retiradas de corpos de prova moldados. As amostras obtidas por extrusão e por moldagem por injeção foram posteriormente preparadas através de crio-ultramicrotomia e observadas através de microscopia eletrônica de transmissão (TEM). Uma "Função Dispersão" foi desenvolvida neste trabalho para comparar as diversas morfologias sob diferentes condições de processamento e de compatibilização. A adição de compatibilizante favorece a formação de uma morfologia de domínios de ABS dispersos em PBT, ao longo do canhão da extrusora, e previne satisfatoriamente o fenômeno de coalescência destes domínios durante o processo de moldagem por injeção. A função dispersão foi utilizada principalmente para mostrar a tendência da evolução morfológica e mostrou um bom desempenho para tal.
\end{abstract}

Palavras-chave: Blendas poliméricas, misturas poliméricas, $P B T / A B S$, extrusão reativa, análise morfológica, microscopia eletrônica de transmissão.

\section{Analysis of the Morphological Development of PBT/ABS Blends during the Extrusion and Injection Molding Processes}

Abstract: The aim of this work was to observe the development of the phase morphology of the PBT/ABS blends during their extrusion mixing and injection molding steps. The evolution of the blend morphology during the mixing stage was analyzed using a specially designed co-rotational twin-screw extruder with a collecting device located along the barrel. Blend samples were collected in-line along the length of the extruder barrel during the blending process. Blend morphology was also observed from specimens molded through injection molding. All the samples were observed by transmission electron microscopy (TEM). They were prepared by cryo-ultramicrotomy and the rubbery phase of the ABS contained in the blends was stained with osmium tetroxide $\left(\mathrm{OsO}_{4}\right)$. A special function was established and is proposed to analyze the blend morphology trends through image analysis. In binary blends, the ABS phase showed better dispersion after the mixture passed through the extruder die. However, a coalescence phenomenon was observed during the next molding step. Compatibilized PBT/ABS blends showed better dispersion than binary blends, even after injection molding. The special function used to quantify the dispersion throughout the mixing and molding steps showed an excellent performance.

Keywords: Polymer blends, PBT/ABS, reactive extrusion, morphology analysis, transmission electron microscopy.

\section{Introdução}

As blendas poliméricas são importantes materiais que se destacam pela ampla faixa de razão custo/benefício. Suas propriedades físicas e químicas podem ser alteradas para uma vasta gama de aplicações, proporcionando desempenho desejável para o produto final. O desenvolvimento de uma blenda polimérica é muitas vezes uma excelente alternativa para o setor de seleção de materiais polimé- $\operatorname{ricos}^{[1,2]}$. Embora um número significativo de blendas poliméricas seja caracterizado como miscível, a maioria das misturas de polímeros é imiscível ${ }^{[3]}$. Geralmente as blendas poliméricas imiscíveis são compatibilizadas ${ }^{[1]}$, para atingirem um desempenho satisfatório. Essas blendas são muitas vezes designadas como Ligas Poliméricas, em analogia ao termo Metal Alloys utilizada na metalurgia.

O processamento de blendas poliméricas consiste basicamente de duas etapas principais: a de mistura ou preparação

Autor para correspondência: Luiz A. Pessan, Departamento de Engenharia de Materiais, UFSCar, Caixa Postal 676, CEP: 13565-905, São Carlos, SP. Email:pessan@power.ufscar.br 
Tabela 1. Dados técnicos fornecidos pelo fabricante

\begin{tabular}{ccccc}
\hline Material & $\begin{array}{c}\text { Resistência à tração } \\
\text { na ruptura } \\
(\mathbf{M P a})\end{array}$ & $\begin{array}{c}\text { Alongamento na } \\
\text { ruptura } \\
(\mathbf{\%})\end{array}$ & $\begin{array}{c}\text { Módulo sob Flexão } \\
(\mathbf{M P a})\end{array}$ & $\begin{array}{c}\text { Índice de Fluidez } \\
(\mathbf{g} / \mathbf{1 0 m i n})\end{array}$ \\
\hline Valox 325 & 48 & 208 & 2445 & $11,0\left(250^{\circ} \mathrm{C} / 1,2 \mathrm{Kg}\right)$ \\
ABS GPM5500 & 50 & 18 & 2400 & $11,0\left(230^{\circ} \mathrm{C} / 5 \mathrm{Kg}\right)$ \\
\hline
\end{tabular}

e a de moldagem ou conformação. Ambas etapas influenciam nas propriedades finais da blenda polimérica ${ }^{[4]}$. As blendas poliméricas são comumente preparadas por misturas mecânicas no estado fundido. As propriedades são determinadas entre outras pela morfologia final, principalmente pela dispersão das fases presentes. Na etapa de mistura ou de preparação da blenda polimérica ocorre a incorporação dos componentes poliméricos, na qual se procura atingir o estado de mistura dese$\mathrm{jado}^{[4]}$. Por outro lado, em quase todos os processos de moldagem ou de conformação utiliza-se a blenda polimérica no estado fundido. Assim, quando a blenda é moldada no estado fundido pode perder algumas das características obtidas durante o processo de mistura por extrusão. Aspectos importantes como: degradação térmica, propriedades reológicas, orientação molecular sob fluxo, e solidificação dos componentes poliméricos podem proporcionar mudanças morfológicas significativas. Portanto, é desejável que a blenda mantenha sua estabilidade morfológica após o processo de moldagem para proporcionar propriedades finais desejadas ${ }^{[4]}$.

A motivação que gerou este trabalho foi devido aos sucessos obtidos na tenacificação da blenda binária PBT/ABS compatibilizada com um copolímero acrílico reativo ${ }^{[5-7]}$ e a necessidade de melhor esclarecer os mecanismos de mudanças de morfologia que ocorrem durante o processo de mistura por extrusão e moldagem por injeção. Assim, procurou-se acompanhar o desenvolvimento da morfologia da referida blenda ao longo da sua etapa de mistura dentro da extrusora, após a saída da extrusora na forma de grânulos e após a moldagem da blenda por injeção. A blenda PBT/ABS teve sua morfologia observada com e sem a incorporação de compatibilizante.

\section{Materiais e Métodos}

\section{Materiais}

Foram utilizados na formulação da blenda: o poli(tereftalato de butileno) (PBT), conhecido pelo nome comercial de Valox 325F, da GE Plastics; o terpolímero de acrilonitrila-butadienoestireno (ABS), conhecido como Cycolac GPM5500S Natural 1000, da GE Plastics, dados técnicos dos materiais comerciais são apresentados na Tabela 1, e como compatibilizante reativo, o poli(metacrilato de metila-co-metacrilato de glicidila) (MMA-GMA), que obteve sucesso na compatibilização reativa da blenda polimérica $\mathrm{PBT} / \mathrm{ABS}$, no trabalho de Mantovani ${ }^{[5]}$. Neste trabalho as concentrações em peso foram de $60 \%$ de PBT, $40 \%$ de ABS e variando de 0,2 e $5 \%$ o copolímero MMA-GMA na composição de $88 \%$ metacrilato de metila (MMA), $10 \%$ metacrilato de glicidila (GMA) e $2 \%$

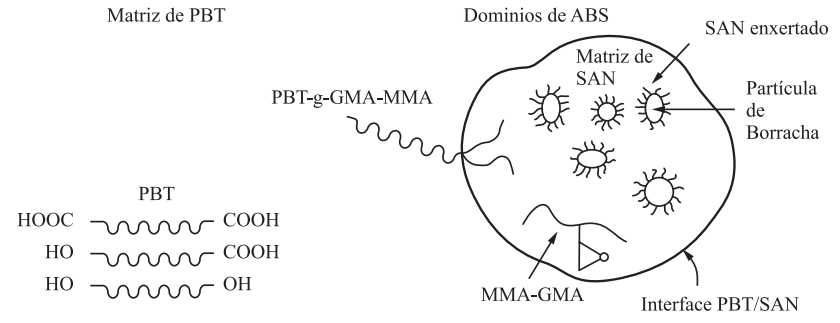

Figura 1. Representação esquemática da morfologia da blenda PBT/ABS compatibilizado pelo copolímero MMA-GMA.

de acrilato de etila (EA). O copolímero MMA-GMA foi sintetizado devido a não ser um produto comercial facilmente encontrado, sendo que o MMA é miscível com o copolímero SAN do ABS e os grupos epóxi, existentes na cadeia do polímero GMA, reagem rapidamente com os grupos carboxilas e hidroxilas do final da cadeia do polímero PBT formando a interface (PBT/SAN) dos polímeros imiscíveis PBT e ABS. Uma pequena quantidade de EA foi adicionada ao copolímero para prevenir a abertura de cadeias ("unzipping") que comumente ocorrem com polímeros de acrílicos em elevadas temperaturas.

A microestrutura esquemática da morfologia da blenda PBT/ ABS/MMA-GMA pode ser representada como na Figura 1, no qual verificamos que a blenda polimérica é formada por uma matriz de PBT com domínios de ABS dispersa. Os domínios de ABS são formados por uma matriz de SAN livre, sendo que uma porcentagem do SAN encontra-se enxertado na sua superfície das partículas de polibutadieno. Como os polímeros PBT e o copolímero SAN são imísciveis, a blenda forma uma interface PBT/SAN. Para compatibilizar essa blenda utilizou-se o copolímero aleatório MMA-GMA, sendo que o polímero PMMA é miscível no polímero SAN e o GMA possui um grupo epóxi funcional na sua estrutura que reage com os grupos carboxilas e hidroxilas dos finais de cadeias do PBT.

\section{Processamento das Blendas}

O processamento das blendas poliméricas foi realizado em uma extrusora, da Leistritz Universal LSM 30.34 GL, de rosca dupla co-rotacional $(\mathrm{L} / \mathrm{D}=29)$, com vários pontos de coleta de amostras ao longo da rosca como pode ser visualizada na Figura 2. Esse método de coleta de amostra, com tempo de coleta menor que 5 segundos usando o conceito de desvio, foi desenvolvido e patenteado por Covas ${ }^{[8]}$. $\mathrm{O}$ aparato pode ser inserido entre quaisquer dois segmentos do canhão da extrusora e proporciona a possibilidade de obter amostras de polímeros com um pequeno aumento de distância 


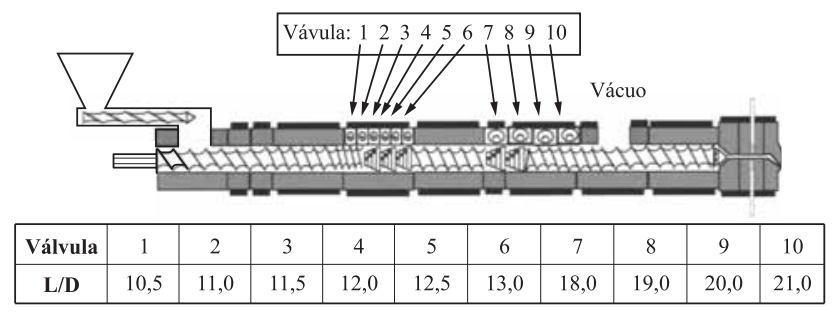

Figura 2. Desenho esquemático do perfil de rosca e os pontos de coletas das amostras ao longo do canhão de extrusão.

ao longo da rosca. Isto é particularmente relevante no estudo da evolução da morfologia em processos de extrusão reativa ao longo da rosca. A configuração da extrusora usada neste trabalho (ver Figura 2) possui ao longo da rosca 10 pontos de coleta de material, sendo esses pontos de coleta projetado na região de intensa mistura. O perfil de rosca utilizado possui elementos de transporte, dois blocos de malaxagem com ângulos de $-60^{\circ}$ (passo esquerdo) e um elemento de transorte passo esquerdo. O perfil de temperatura de processamento foi fixada em $240{ }^{\circ} \mathrm{C}$ em todas as zonas e foi utilizada uma vazão de $4 \mathrm{Kg} / \mathrm{h}$.

A mistura PBT/ABS/MMA-GMA, por extrusão, foi realizada dosando-se simultaneamente os componentes no funil de alimentação da extrusora. À medida que ambos materiais fundiam, iniciava-se o processo efetivo de mistura ao longo do canhão. A coleta de amostras desta mistura foi realizada conforme ilustrado na Figura 2.

Nas primeiras válvulas de coleta, Figura 2, número 1 e 2, não foi possível realizar coletas das amostras, pois a mistura não se encontrava totalmente fundida e/ou não havia pressão suficiente nestes pontos de coleta para empurrar o material fundido através das válvulas. A coleta das amostras só foi possível a partir da válvula 3 , pois os blocos de malaxagem proporcionavam condições suficientes para fusão dos materiais e geração de pressão interna para expulsão da mistura. As amostras coletadas na válvula 3 apresentaram uma morfologia variável, isto é, totalmente irregulares, apresentando ora regiões com grande concentração de PBT e ora outras com grandes concentrações de ABS. As morfologias puderam ser melhores observadas para amostras retiradas a partir da válvula 4 até a válvula 8 . Nas válvulas 9 e 10 também não foi possível realizar a retirada das amostras devido o ponto de coleta estar após um bloco de condução de passo esquerdo, portanto sem a pressão suficiente para retirar as amostras. Ao final do canhão as misturas passaram por uma matriz utilizada para sua granulação, após a qual suas morfologias foram caracterizadas. As misturas granuladas foram então moldadas por injeção na forma de corpos de prova para ensaios de impacto Izod.

\section{Métodos}

As amostras coletadas ao longo do canhão de extrusão, as coletadas na saída da matriz e as injetadas na forma de corpo de prova de impacto, foram catalogadas e preparadas através do procedimento de redução de área transversal da amostra ("trimming") e o dimensionamento da ponta da amostra, a ser ultramicrotomada em forma trapezoidal, que proporciona uma melhor distribuição de tensão no corte de filmes, com área superficial aproximadamente de $1,0 \mathrm{~mm}^{2}$.

Posteriormente realizaram-se os cortes das amostras, em um ultramicrótmo da marca RMC MT-7000 e um outro da marca Leica, modelo Reichert Ultracut S, usando facas de diamante da marca Diatome tipo CryoHisto $45^{\circ}$, com a temperatura de corte de $-60^{\circ} \mathrm{C}$, com resfriamento em nitrogênio líquido, para evitar a deformação da microestrutura original. A velocidade de corte foi de $0,1 \mathrm{~mm} / \mathrm{s}$ e espessura da amostra de $50 \mathrm{~nm}$.

As amostras ultramicrotomadas foram colocadas sobre o vapor de tetróxido de ósmio, por 15 horas, para que ocorresse a incorporação de átomos de alto número atômico na fase borrachosa. Neste caso o tetróxido de ósmio ataca a insaturação do polibutadieno proporcionando um aumento da densidade na fase borrachosa e, conseqüentemente, um maior contraste entre os domínios nas análises realizadas por microscopia eletrônica de transmissão (TEM), a qual se deu em um equipamento de Philips, modelo CM120, com uma tensão de $120 \mathrm{kV}$.

\section{Resultados e Discussão}

\section{Caracterização Química do ABS}

Na Tabela 2 são apresentados os resultados das análises de caracterização da resina $\mathrm{ABS}$ utilizada neste trabalho. Dentre as técnicas utilizadas para a caracterização da resina ${ }^{[9]}$, destacam-se as de quantificação de: teor dos meros, massa molar do SAN livre, porcentagem de gel, índice de enxertia (IG), eficiência de enxertia (EG) e densidade de enxertia (DG). A porcentagem de gel é a quantidade de copolímero SAN enxertado na partícula de polibutadieno. A determinação da porcentagem de gel foi obtida por ultracentrifugação. Quantitativamente, o índice de enxertia (IG) é a quantidade em massa de SAN enxertado por $100 \mathrm{~g}$ de polibutadieno. A eficiência de enxertia (EG) é a quantidade em massa de SAN enxertado por $100 \mathrm{~g}$ de meros do copolímero SAN total, incluindo SAN livre e enxertado. A densidade de enxertia (DG) é o número médio de "grafts" por unidade monomérica de polibutadieno, sendo que, o inverso da densidade de enxertia representa quantitativamente a distância entre dois "grafts".

A porcentagem em massa de acrilonitrila no ABS foi determinada pela média de três medidas obtidas por análise elementar CHNS-O e por FTIR. A porcentagem em massa de butadieno e a de estireno foi determinada pela da média de três análises de FTIR. Utilizou-se de um espectrômetro FTIR, que através de procedimentos de calibração e constantes cálculados por Mantovani ${ }^{[5]}$, realizou-se os cálculos utilizando os picos de absorbâncias das bandas em 2237 $\mathrm{cm}^{-1}, 1490 \mathrm{~cm}^{-1}$ e $965 \mathrm{~cm}^{-1}$, referente aos picos característicos do acrilonitrila, estireno e butadieno, respectivamente.

As massas molares numéricas médias $\left(\overline{M_{n}}\right)$ e ponderal média $\left(\overline{M_{w}}\right)$, do SAN livre que foi extraído por ultracentrifugação do ABS, foram determinadas por SEC. 
Tabela 2. Composição química e caracterização molecular do ABS

\begin{tabular}{|c|c|c|c|c|}
\hline \multirow{2}{*}{$\begin{array}{c}\text { Material } \\
\text { ABS - Cycolac } \\
\text { GPM5500S }\end{array}$} & $\begin{array}{l}\text { Gel } \\
(\%)\end{array}$ & $\begin{array}{l}\mathrm{IG} \\
(\%)\end{array}$ & $\begin{array}{l}\text { EG } \\
(\%)\end{array}$ & $\begin{array}{c}\mathrm{DG}^{(\mathrm{a})} \\
\text { (unidades) }\end{array}$ \\
\hline & 27,3 & 50,8 & 11,2 & $2,19 \times 10^{-4}$ \\
\hline \multirow{2}{*}{$\begin{array}{l}\text { Composição } \\
\text { (\% em massa) }\end{array}$} & \multicolumn{2}{|c|}{ Acrilonitrila } & Butadieno & Estireno \\
\hline & $22,5^{(\mathrm{c})}$ & \multicolumn{2}{|c|}{$18,1^{(\mathrm{c})}$} & $59,4^{(\mathrm{c})}$ \\
\hline \multirow{2}{*}{$\begin{array}{c}\text { Massa molar } \\
\text { SAN Livre }(\mathrm{g} / \mathrm{mol})\end{array}$} & \multicolumn{2}{|c|}{$\overline{M_{n}}$} & \multicolumn{2}{|c|}{$\overline{M_{w}}$} \\
\hline & \multicolumn{2}{|c|}{64194} & \multicolumn{2}{|c|}{125324} \\
\hline
\end{tabular}

IG - Índice de enxertia; EG - Eficiência de enxertia; DG - Densidade de enxertia.

(a) A unidade do DG foi medida como número médio de "grafts"por unidade monomérica de butadieno, como definido por Chauvel e Daniel ${ }^{[9]}$.

(b) Determinado por análise elementar CHNS-O.

(c) Determinado por FTIR

Através dos resultados obtidos verifica-se que a composição do ABS possui uma menor quantidade de fase elastomérica e uma maior quantidade do comonômero estireno, ou seja, este tipo de ABS tem como característica uma maior facilidade de processamento e uma resistência ao impacto não muito elevado, como será mostrado no item de caracterização mecânica dos materiais. Com relação à fase de SAN enxertado em polibutadieno, observa-se que aproximadamente $11,2 \%$ em massa do copolímero SAN encontra-se enxertado na fase elastomérica e aproximadamente $88,8 \%$ do SAN encontra-se livre como matriz do ABS. O IG mostra que para cada 100 gramas de polibutadieno existem aproximadamente 50 gramas de SAN enxertado. O nível de enxertia influencia diretamente no tamanho das partículas da fase elastomérica e a sua distribuição na matriz do ABS. Conseqüentemente, o estado morfológico da fase dispersa contribui significativamente para a tenacidade do ABS. A massa molar do SAN livre extraído, ou seja, não enxertado, é mostrada na Tabela 2. Este parâmetro influencia diretamente o estado de miscibilidade das moléculas de SAN com o copolímero MMA-GMA utilizado como compatibilizante.

O nível de enxertia influencia diretamente no tamanho das partículas da fase elastomérica e a sua distribuição na matriz do ABS, assim, influenciando diretamente nas propriedades finais da blenda polimérica.

\section{Caracterização Termo-dinâmico-mecânica do PBT, ABS, e das blendas poliméricas}

A caracterização térmica dos materiais é importante tanto do ponto de vista de processabilidade como do ponto de vista do comportamento termo-mecânico. A análise termodinâmico-mecânica mede a alteração do módulo de elasticidade do material em função da temperatura. Uma queda brusca do módulo elástico dinâmico (E') ou um pico acentuado do módulo viscoso (módulo de perda) (E") indica a temperatura onde ocorre a transição vítrea ${ }^{[4]}$. A Figura 3 mostra as curvas de E' e de $\tan \delta$ versus temperatura para o polímero PBT, o terpolímero ABS e para as blendas poliméricas $\mathrm{PBT} / \mathrm{ABS}$ com 0,2 e $5 \%$ do copolímero MMA-GMA.

A Figura 3(a) mostra que o PBT apresenta uma transição principal em torno de $60^{\circ} \mathrm{C}$, correspondente à transição vítrea da sua fase amorfa e outra transição secundária $\beta$ em torno de $-76^{\circ} \mathrm{C}$, correspondente aos movimentos tipo "crankshaft" em sua cadeia polimérica principal. O comportamento termomecânico do ABS pode ser observado na Figura 3(b). Por ser um sistema multifásico o ABS apresenta transições correspondentes à transição vítrea da fase elastomérica em torno de $-73{ }^{\circ} \mathrm{C}$ e da transição vítrea da fase matriz SAN em torno de $109^{\circ} \mathrm{C}$. A intensidade do pico de $\tan \delta$ para a fase borrachosa é muito inferior à do pico da fase SAN, pois esta é fase minoritária no sistema. A Figura 3(c), da blenda binária PBT/ $\mathrm{ABS}$, apresenta transições vítreas em $-68,60$ e a $100^{\circ} \mathrm{C}$, referente as transições da fase elastomérica, do PBT e do SAN, respectivamente. A blenda polimérica PBT/ABS/MMA-GMA pode ser analisada na Figura 3(d), no qual apresenta transições em -66, 60 e a $98^{\circ} \mathrm{C}$. Enfim, pode ser constatado através da análise termo-dinâmico-mecânica que os materiais apresentam transições muito bem definidas. Este trabalho, assim como em trabalhos anteriores ${ }^{[5,7,11]}$, mostra que PBT e ABS formam blendas poliméricas totalmente imiscíveis, quando analisadas sob o critério de temperatura de transição vítrea.

\section{Caracterização Reológica dos Materiais Utilizados}

No processamento de polímeros, as propriedades reológicas dependem dos parâmetros operacionais (temperatura, pressão, vazão, etc) e estruturais (massa molar, distribuição de massa molar, etc). Assim, é recomendável medir propriedades, como a viscosidade, nas condições mais próximas às condições de processamento ${ }^{[10]}$.

Hale et al. ${ }^{[1]}$ estudaram o efeito da variação da viscosidade do PBT, no estado fundido, na morfologia de blendas PBT com ABS e/ou SAN e verificaram que a morfologia e as propriedades de fratura são sensíveis ao tipo de PBT utilizado. Os melhores resultados foram obtidos para o PBT com maior viscosidade onde ocorreu uma melhor dispersão dos domínios de ABS e/ou SAN.

\section{Caracterização através de Reometria Capilar}

A reometria capilar é a técnica experimental mais comum 


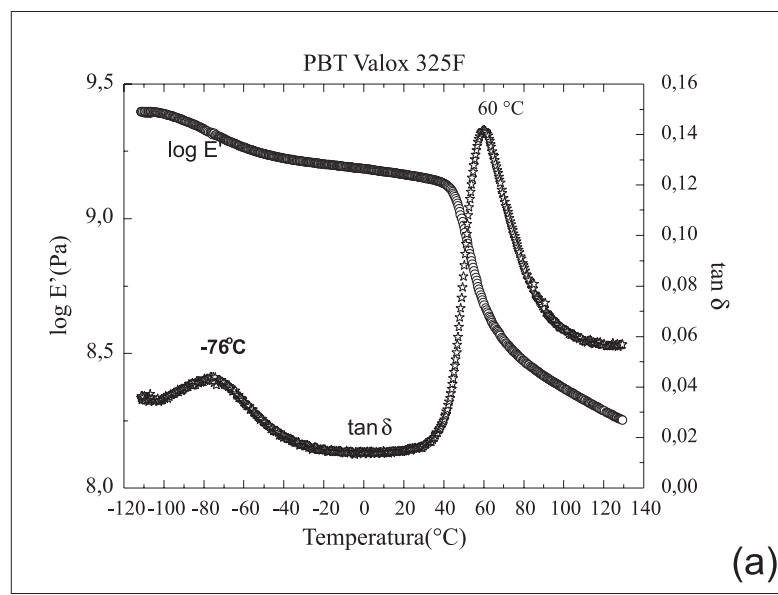

(a)

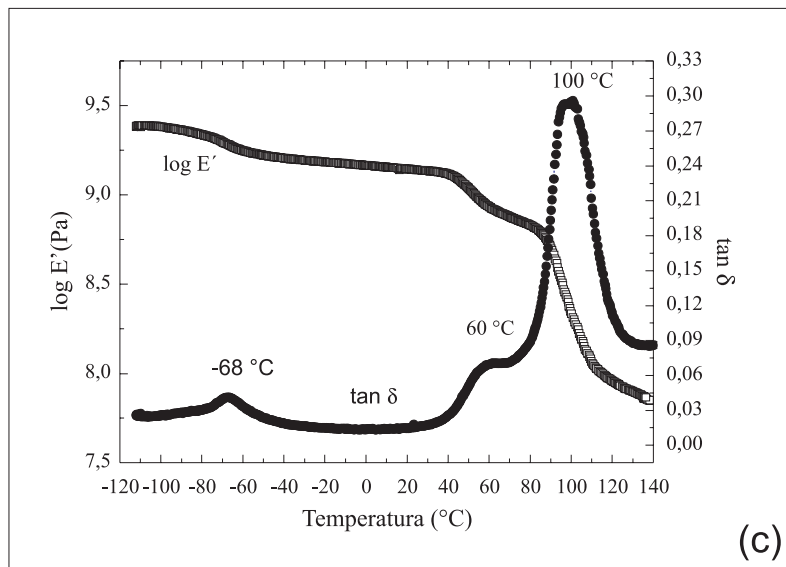

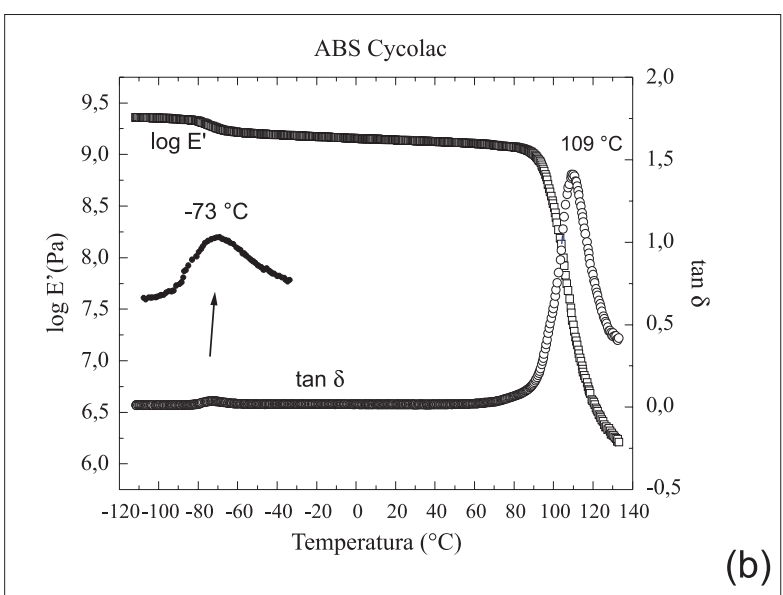

(b)

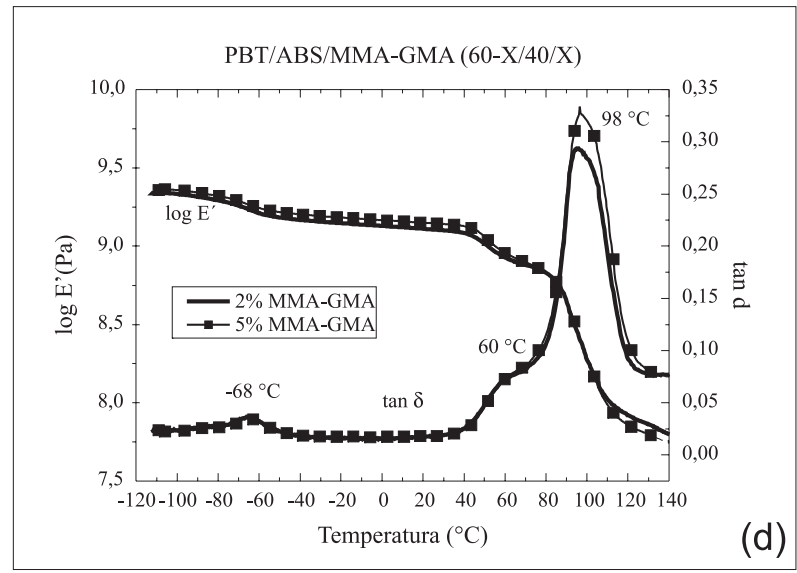

Figura 3. Análise termo-dinâmico-mecânica do polímero PBT (Valox 325F) (a); do terpolímero ABS (Cycolac GPM5500S) (b); da blenda binária PBT/ ABS (60/40) (c); e da blenda polimérica PBT/ABS/MMA-GMA com 2 e 5\% de MMA-GMA (d), realizada a uma taxa de aquecimento de $3^{\circ} \mathrm{C} / \mathrm{min}$ e uma freqüência de $1 \mathrm{~Hz}$.

para medidas das propriedades reológicas de polímeros fundidos sob fluxo viscoso ${ }^{[12,13]}$. O escoamento viscoso num capilar é um exemplo de fluxo parcialmente controlável; ou seja, longe da sua entrada, onde o fluxo se torna completamente desenvolvido, as linhas de fluxo são sempre paralelas ao eixo do tubo. Por outro lado, o perfil de velocidade dependerá da natureza reológica do fluido. As medidas feitas com reômetros capilares estão limitadas a taxas de cisalhamento acima de $10^{-1} \mathrm{~s}^{-1}$, portanto, esta é uma técnica bastante útil para avaliar a viscosidade do material durante o processamento por extrusão (taxas de cisalhamento entre 100 a $1000 \mathrm{~s}^{-1}$ ) e moldagem por injeção (taxas de cisalhamento entre 1000 a $10000 \mathrm{~s}^{-1}$ ).

A Figura 4 (a, b e c) apresenta o comportamento reológico, através de reometria capilar, dos materiais poliméricos estudados, utilizando-se de um capilar com L/D de 33, nas temperaturas de $240^{\circ}, 250^{\circ}$ e $260^{\circ} \mathrm{C}$, respectivamente. Verifica-se que o comportamento reológico dos polímeros utilizados apresenta viscosidades com valores próximos até uma taxa de cisalhamento em torno de $400 \mathrm{~s}^{-1}$. A partir desta taxa de cisalhamento as curvas divergem indicando uma maior viscosidade para o PBT em relação ao ABS. O comportamento é semelhante para cada uma das temperaturas utilizadas, como podemos verificar através dos dados de razão de viscosidade apresentados na Tabela 3.

\section{Caracterização através de Reometria de Torque}

Ensaios reológicos realizados através de reometria de torque à temperatura de $240{ }^{\circ} \mathrm{C}$ e rotação de $50 \mathrm{rpm}$ são apresentados na Figura 5. Através das curvas apresentadas, comparam-se os comportamentos do polímero PBT puro e do terpolímero ABS puro em relação a adição nos mesmos do compatibilizante utilizado na blenda polimérica.

A Figura 5(a) mostra o indício da reação entre o grupo epóxi do GMA com os finais de cadeias do PBT. Pois, no decorrer do tempo de mistura ocorreu o aumento de torque, o que inclui o aumento da viscosidade com o aumento de massa molar das cadeias poliméricas de PBT gerada pela reação. A incorporação de 5\% em massa de MMA-GMA proporciona um aumento de torque da mistura PBT/MMA-GMA, a uma taxa constante. A reação é tão intensa mostrando indício da ocorrência de um certo grau de reticulação entre as moléculas do PBT e do MMA-GMA.

Em trabalhos anteriore ${ }^{[5]}$ com a blenda PBT/ABS foi verificado que o emulsificante residual, gerado no processo de polimerização por emulsão do $\mathrm{ABS}$, ativa um processo de reação de degradação do PBT através de transesterificação. Geralmente os emulsificantes são ácidos graxos com elevado poder de reação. Além disso, a presença de resíduos de 


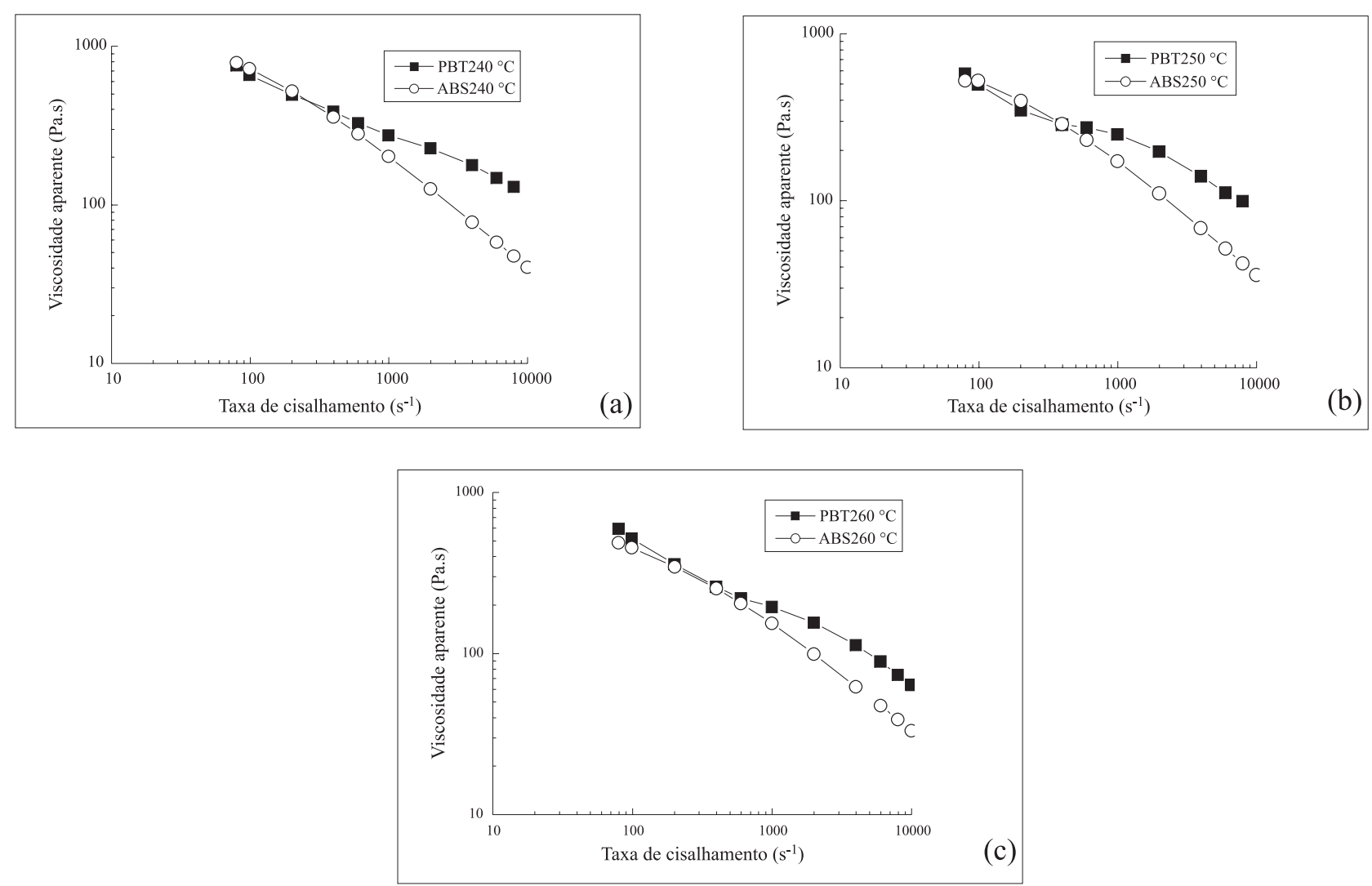

Figura 4. Reometria Capilar do PBT - Valox 325F e do ABS - Cycolac GPM5500S nas temperaturas: (a) $240^{\circ}$; (b) $250^{\circ}$ e (c) $260^{\circ} \mathrm{C}$ e um capilar com (L/D $=33$ ).

Tabela 3. Razão de Viscosidade entre os polímeros ABS e PBT obtidas por reometria capilar a temperaturas de $240^{\circ}, 250^{\circ}$ e $260^{\circ} \mathrm{C}$.

\begin{tabular}{|c|c|c|c|c|}
\hline Polímeros & $\begin{array}{c}\text { Temperatura } \\
\left({ }^{\circ} \mathbf{C}\right) \\
\end{array}$ & $\begin{array}{c}\text { Taxa de Cisalhamento } \\
\left(\mathrm{s}^{-1}\right)\end{array}$ & $\begin{array}{c}\text { Viscosidade Aparente } \\
\text { (Pa.s) }\end{array}$ & $\begin{array}{l}\text { Razão de Viscosidade } \\
\left(\eta_{\mathrm{ABS}} / \eta_{\mathrm{PBT}}\right)\end{array}$ \\
\hline $\begin{array}{l}\text { ABS } \\
\text { PBT }\end{array}$ & 240 & 400 & $\begin{array}{l}359,88 \\
388,44\end{array}$ & 0,93 \\
\hline $\begin{array}{l}\text { ABS } \\
\text { PBT }\end{array}$ & 240 & 1000 & $\begin{array}{l}202,09 \\
275,16\end{array}$ & 0,73 \\
\hline $\begin{array}{l}\text { ABS } \\
\text { PBT }\end{array}$ & 240 & 4000 & $\begin{array}{c}77,95 \\
178,77\end{array}$ & 0,44 \\
\hline $\begin{array}{l}\text { ABS } \\
\text { PBT }\end{array}$ & 250 & 400 & $\begin{array}{l}288,70 \\
286,20\end{array}$ & 1,01 \\
\hline $\begin{array}{l}\text { ABS } \\
\text { PBT }\end{array}$ & 250 & 1000 & $\begin{array}{l}172,87 \\
251,17\end{array}$ & 0,69 \\
\hline $\begin{array}{l}\text { ABS } \\
\text { PBT }\end{array}$ & 250 & 4000 & $\begin{array}{c}68,58 \\
140,76\end{array}$ & 0,49 \\
\hline $\begin{array}{l}\text { ABS } \\
\text { PBT }\end{array}$ & 260 & 400 & $\begin{array}{l}254,02 \\
260,18\end{array}$ & 0,98 \\
\hline $\begin{array}{l}\mathrm{ABS} \\
\mathrm{PBT}\end{array}$ & 260 & 1000 & $\begin{array}{l}154,38 \\
195,21\end{array}$ & 0,79 \\
\hline $\begin{array}{l}\text { ABS } \\
\text { PBT }\end{array}$ & 260 & 4000 & $\begin{array}{c}62,51 \\
113,39\end{array}$ & 0,55 \\
\hline
\end{tabular}

polimerização do ABS pode comprometer a compatibilização reativa in situ promovida pelo MMA-GMA entre PBT e $\mathrm{ABS}^{[5]}$, diminuindo a sua eficiência de compatibilização da blenda. Muitas vezes a reometria de torque é utilizada para mostrar evidências destas possíveis reações colaterais ${ }^{[14]}$. Um aumento significativo ou uma queda brusca de torque da mistura, em relação aos componentes puros, indica a ocorrência de reações indesejadas. No caso da mistura de ABS/MMAGMA, Figura 5 (b), foi possível observar, através deste critério, que os resíduos de polimerização do ABS favorece 
um ligeiro aumento do torque da mistura. Os valores de torque do sistema ABS/MMA-GMA mostraram-se superiores ao do ABS puro, indicando a ocorrência de algum tipo de reação, gerando o aumento da crescimento de massa molar. Mantovani ${ }^{[5]}$ registrou um aumento significativo de torque para a mistura ABS/MMA-GMA, em relação ao ABS utilizado, e mostrou que resíduos de emulsificante provocam a reticulação do copolímero MMA-GMA, tornando-o completamente termorrígido. Por outro lado, se o ABS fosse polimerizado em massa não haveria ácidos residuais, portanto, não ocorreria a reação na mistura polimérica, como foi verificado por Hale et al. ${ }^{[14,15]}$.

A utilização de MMA-GMA como compatibilizante para o sistema PBT/ABS está baseada no princípio de modificação da região interfacial entre as fases PBT e ABS. Esta modificação consiste na reação in situ do grupo funcional epóxi existente no comonômero GMA do MMA-GMA com os finais de cadeia do PBT, principalmente os grupos carboxílicos. O restante dos segmentos moleculares do copolímero (MMA) é miscível com a fase rica em SAN do ABS, como estudado por Gan e Paul ${ }^{[17]}$. Assim, moléculas de PBT enxertadas com moléculas de MMA-GMA tornam-se agentes compatibilizantes, reduzindo o tamanho de domínios da fase dispersa e aumentando a adesão interfacial entre as fases da blenda polimérica. Para tanto, é necessário observar a ocorrência da reação entre PBT e o copolímero MMA-GMA. A reometria de torque foi utilizada para observar as evidências desta reação. Portanto, um aumento do torque, durante a mistura PBT/ MMA-GMA, é sinal do aumento de massa molar decorrente da reação de enxertia de ambos componentes.
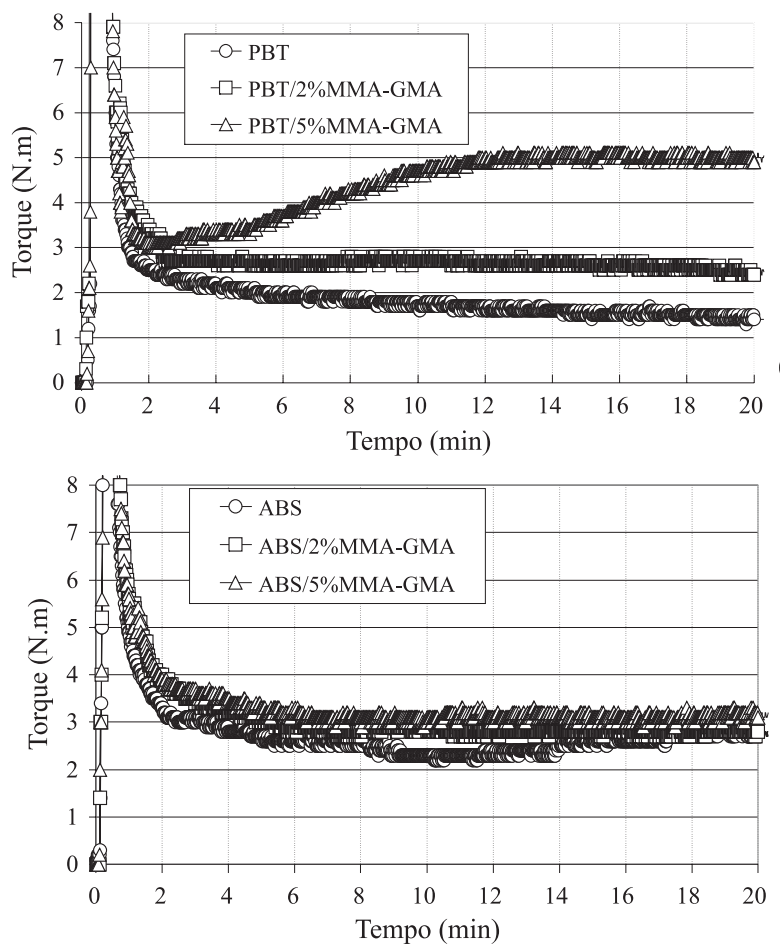

Figura 5. Curvas de torque em função do tempo, por 20 minutos, obtidas no HAAKE a $240{ }^{\circ} \mathrm{C}$ e $50 \mathrm{rpm}$ para o polímero PBT, para o terpolímero ABS e suas blendas com $2 \%$ e $5 \%$ de MMA-GMA.

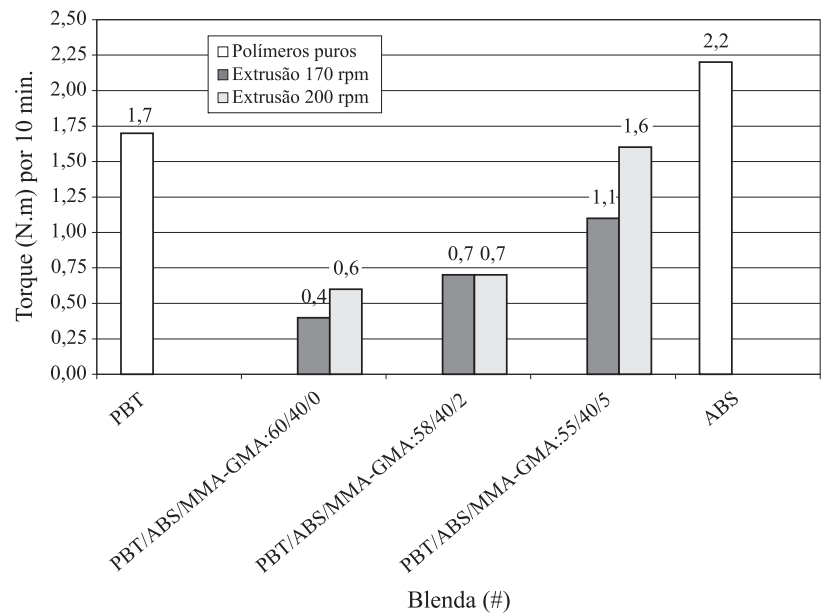

Figura 6. Reometria de Torque por 10 minutos dos polímeros $\mathrm{PBT}$, ABS e das blendas PBT/ABS/MMA-GMA com composição 60-X/40/X, a temperatura de $240{ }^{\circ} \mathrm{C}$, a velocidade de $50 \mathrm{rpm}$.

Na Figura 6 são apresentados os resultados de reometria de torque para as blendas poliméricas PBT/ABS processadas por extrusão a $240{ }^{\circ} \mathrm{C}$, sob diferentes velocidades de rotação das roscas. Os resultados mostram que as blendas poliméricas previamente misturadas por extrusão apresentam comportamento diferenciado quando submetidas a fluxo em um reômetro de torque. Apesar dos polímeros puros PBT e ABS não possuírem a mesma história térmica, pois as blendas foram submetidas a uma etapa de processamento por extrusão a mais do que os polímeros puros, os valores de torque são sensivelmente superiores aos das blendas, principalmente com a incorporação do copolímero compatibilizante. Na temperatura utilizada no HAAKE de $240{ }^{\circ} \mathrm{C}$, os valores de torque para as blendas não compatibilizadas se mostraram inferiores aos dos componentes puros. Este comportamento, provavelmente, deve ser resultado da degradação termo-mecânica sofrida por ambos componentes na mistura, principalmente pelo PBT, conforme já mostrado por Hage et al. ${ }^{[4]}$. A presença do copolímero MMA-GMA nas blendas favorece um aumento de torque em função da reação química in situ durante a etapa de mistura e de fluxo no HAAKE, conforme já observado nas Figuras 5a e 5b.

\section{Desenvolvimento Morfológico}

A Figura 7 mostra as seqüências de morfologia para as blendas PBT/ABS/MMA-GMA, apresentando como variáveis de processo: posição ao longo do canhão de extrusão, compatibilização interfacial e temperatura de injeção.

Após passar pela matriz e ser granulada, a mistura PBT/ ABS adquire uma morfologia completamente definida com ABS sendo a fase dispersa e o PBT, a matriz. Este comportamento no processo de preparação da mistura por extrusão indica que o fluxo viscoso, desenvolvido por cisalhamento nos capilares da matriz, é mais eficiente na dispersão do ABS no PBT do que o fluxo de arraste sofrido pela mistura entre os elementos de rosca no canhão. $\mathrm{O}$ efeito da moldagem por injeção proporciona um fenômeno de coalescência na fase dispersa fazendo com que os domínios de ABS voltem a se aglomerar como pode ser observado na Figura 7. Entretanto, o 


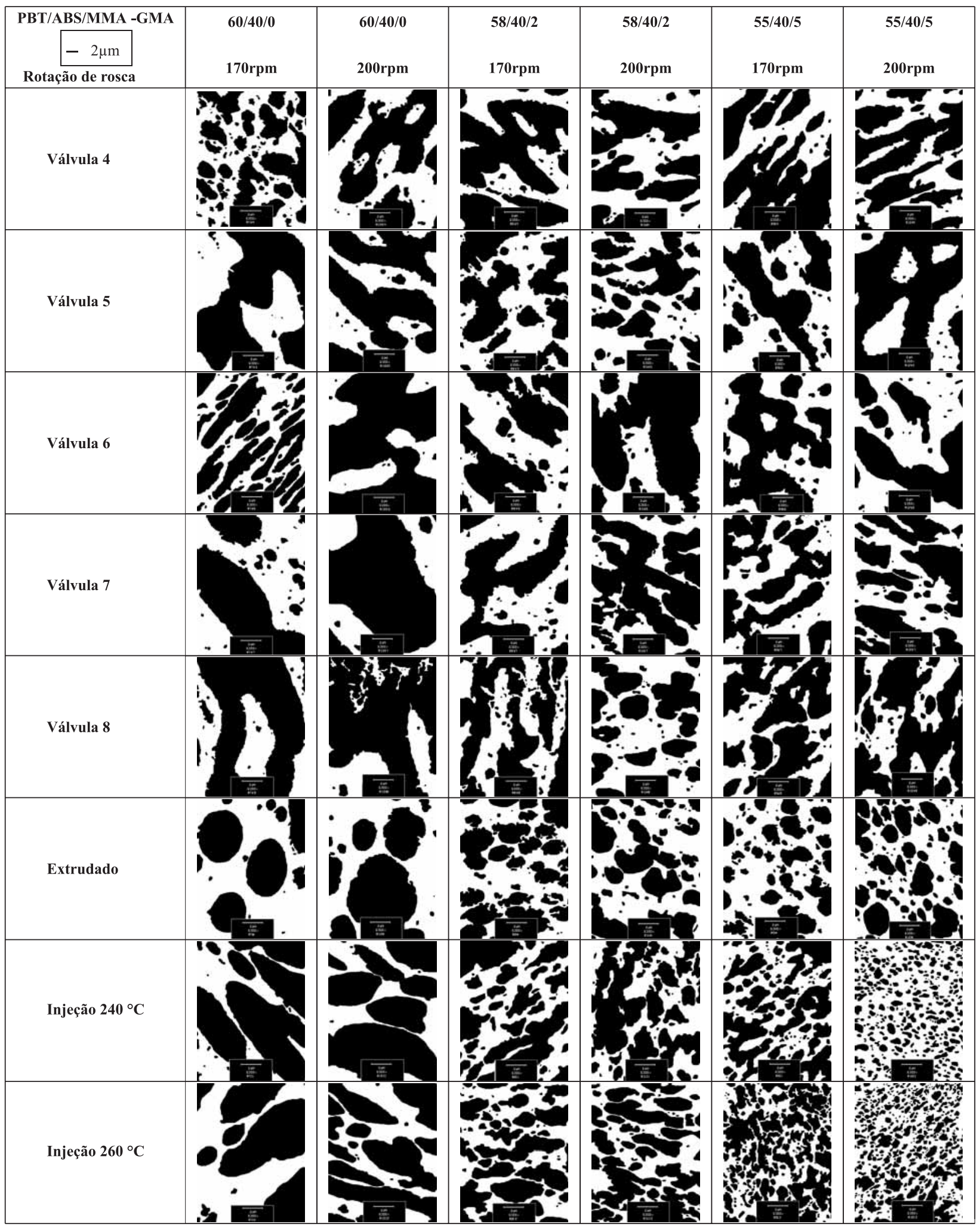

Figura 7. Fotomicrografias da blenda PBT/ABS/MMA-GMA (60-X/40/X) (\%peso), extrudada a 170 e $200 \mathrm{rpm}$ com temperatura de $240{ }^{\circ} \mathrm{C}$, apresentando as regiões escuras formadas pelos domínios de ABS e as regiões claras pela matriz de PBT

mesmo fenômeno de coalescência não é observado durante a moldagem por injeção das amostras com incorporação do copolímero MMA-GMA. Com o aumento da temperatura de injeção ocorre a diminuição dos tamanhos dos domínios de fase dispersa de $\mathrm{ABS}$, mostrando que o copolímero continua reagindo durante o processo de moldagem por injeção.

A incorporação do copolímero MMA-GMA, como compatibilizante para a blenda $\mathrm{PBT} / \mathrm{ABS}$, modifica o comportamento 
de evolução morfológica da blenda. Hale et al. ${ }^{[6]}$ estudaram os efeitos de compatibilização na blenda PBT/ABS com MMAGMA, obtendo resultados satisfatórios de tenacificação com 5\% de MMA-GMA e observaram que com adição maiores não ocorriam mudanças significativas de compatibilização. O compatibilizante proporciona melhor capacidade de dispersão para a blenda e estabiliza a sua morfologia obtida durante a extrusão e durante o processo de moldagem por injeção.

A compatibilização reativa da blenda PBT/ABS realizada através da presença do copolímero MMA-GMA está baseada na reação química in situ do grupo epóxi existente no componente GMA do copolímero e com os grupos químicos carboxílicos e hidroxílicos das extremidades de moléculas do $\mathrm{PBT}^{[6]}$. A cinética deste tipo de reação química depende essencialmente da temperatura e do intervalo de tempo que permite ocorrer este tipo de reação no estado fundido ${ }^{[16]}$. Portanto, o aumento da temperatura de extrusão afeta diretamente a ocorrência da reação através da equação de Arrhenius, enquanto o aumento da rotação diminui o tempo de permanência do fundido na extrusora. Porém, neste último caso, o aumento da rpm contribui com um aumento de calor viscoso, que aumenta a temperatura do fundido. Assim, o efeito de velocidade de rotação de rosca e da temperatura pode estar aumentando a intensidade de reação, favorecendo a modificação interfacial da blenda e dispersando melhor a fase rica em borracha do ABS.

\section{Análise de Imagens}

Devido à ocorrência de possíveis erros gerados na medição dos tamanhos médios equivalentes e das quantidades dos domínios dispersos de ABS, resolveu-se analisar a dispersão dos domínios na matriz de PBT através de uma função especialmente estabelecida para tal (equação 1).

$$
\text { Função Dispersão }=\frac{(\eta)}{\sum \text { AreaTotal }} \times \frac{\sum \text { PerímetroABS }}{\sum \text { AreaABS }}
$$

Esta função, denominada Função Dispersão, procura quantificar a razão entre o número de domínios(n), contado na fotomicrografia, e a área total correspondente. Levando em consideração que os aspectos geométricos dos domínios apresentam formatos irregulares, resolveu-se incluir um termo nesta função para compensar esta irregularidade. Assim, incorporou-se o termo somatório da razão perímetro dos domínios pela área destes domínios.

As fotomicrografias obtidas através da microscopia eletrônica de transmissão foram tratadas para facilitar a análise de imagens. Para tanto, os domínios com tonalidade mais escura foram digitalmente enegrecidas. Esse procedimento proporcionou melhor contraste de fases das fotomicrografias, facilitando o tratamento de imagens, utilizando-se o software Image-Pro Plus versão 3.0 da marca Media Cybernetics.

Até este ponto a análise do desenvolvimento morfológico da blenda PBT/ABS foi realizada através de observações visuais das fotomicrografias. Porém, esta análise pode ser considerada relativamente qualitativa, com forte componente subjetiva. A Função Dispersão foi estabelecida para mostrar a

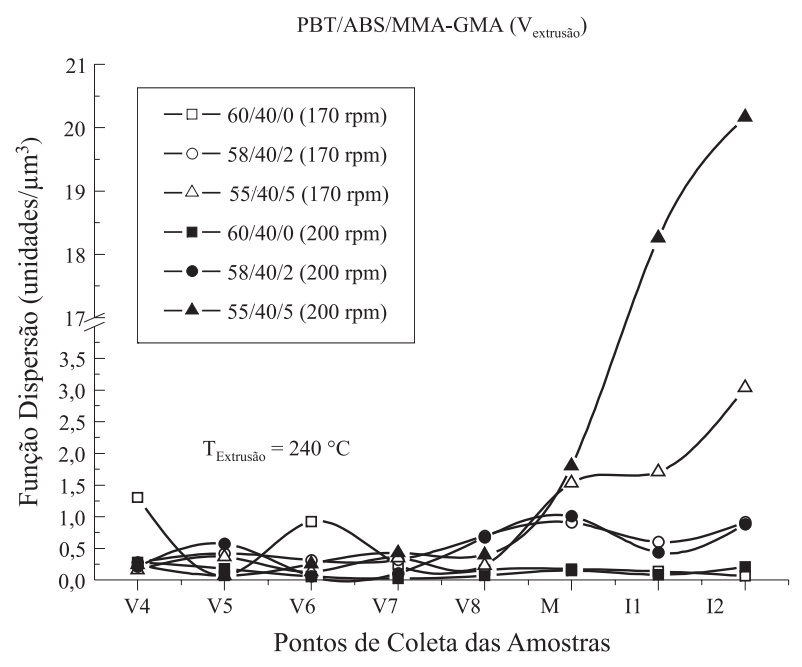

Figura 8. Função Dispersão vs Pontos de Coleta das Amostras para as blendas misturadas por extrusão a temperatura de $240^{\circ} \mathrm{C}$ e rotação de rosca de $170 \mathrm{e}$ $200 \mathrm{rpm}$.

tendência da evolução morfológica da blenda PBT/ABS, durante seu processamento, de uma forma quantitativa. O comportamento da Função Dispersão pode ser observado na Figura 8. Através da Função Dispersão verificamos que para a amostra binária (PBT/ABS) não houve qualquer mudança significativa na tendência da evolução morfológica.

Para as blendas PBT/ABS (60/40) compatibilizadas com $2 \%$ de MMA-GMA, não houve alteração significativa no grau de dispersão da fase rica em $\mathrm{ABS}$, ao longo do canhão de extrusão. Entretanto, para as amostras com 5\% de MMAGMA foi verificado que um aumento na velocidade de rotação das roscas (Figura 8) permitiu um grau de dispersão bastante acentuado somente após a moldagem por injeção. Assim, pode ser concluído que a Função Dispersão foi útil para quantificar a tendência da evolução morfológica durante o processamento da blenda $\mathrm{PBT} / \mathrm{ABS}$.

\section{Conclusão}

O estudo do comportamento morfológico para a blenda binária PBT/ABS mostrou que a blenda é imiscível e suas morfologias são instáveis durante seu processamento.

A presença do compatibilizante torna a microestrutura mais refinada com tamanhos de domínios de ABS menores e mais bem dispersos na matriz de PBT.

A função dispersão, desenvolvida neste trabalho, é útil para mostrar as tendências no desenvolvimento morfológico ao longo do processo de extrusão e após a moldagem por injeção.

\section{Agradecimentos}

Os autores agradecem ao apoio financeiro dado pelas agências CNPq, FAPESP e PRONEx/FINEP/CNPq.

\section{Referências Bibliográficas}

1. Utracki, L. A. - "Polymer Alloys and Blends: Thermodynamics and Rheology", Hanser Publishers, New York (1989). 
2. Utracki, L. A., Shi, Z. H. - Polym. Eng. and Sci., 32, p.1824 (1992).

3. Paul, D. R., Barlow, J. W., Keskkula, H. - "Polymer Blends, In: Encyclopedia of Polymer Science and Engineering", John Wiley \& Sons, New York (1988).

4. Hage, E., Hale, W., Keskkula, H., Paul, D. R. - Polymer, 38, p.3237 (1997)

5. Mantovani, G. L. - “Compatibilização por Extrusão Reativa de Blendas Poliméricas PBT/ABS e Estudo da Estabilização da Morfologia de Fases", Tese de Doutorado, Universidade Federal de São Carlos, Brasil (2002).

6. Hale, W., Keskkula, H., Paul, D. R. - Polymer 40, p.365 (1999).

7. Mantovani, G. L., Canto, L. B., Hage Jr., E., Pessan, L. A. - Macromolecular Symposia 176, P.167 (2001).

8. Machado, A. V., Covas, J. A., Duin, M.Van. - J. Appl. Polym. Sci., 71, p.135 (1999).

9. Chauvel, B., Daniel, J. C. - "Analytical Study of ABS Copolymers Using a preparative Ultracentrifuge.
In:Copolymers, Blends, and Composites", Washington, American Chemical Society (1975).

10. Bretas, R. E. S., D’ávila, M. A. - "Reologia de Polímeros Fundidos”, São Carlos, Editora da UFSCar (2000).

11. Hale, W., Lee, J. H., Keskkula, H., Paul, D. R. - Polymer, 40, p.3621 (1999)

12. Pearson, J. R. A. - "Mechanics of Polymer Processing", New York, Elsevier Applied Science Publishers (1985).

13. Dealy J. M. - "Rheometers for Molten Plastics", New York, Van Nostrand Reinhold Company, (1982).

14. Hale, W., Keskkula, H., Paul, D.R. - Polymer, 40, p.365 (1999).

15. Hale, W. R., Pessan, L. A., Keskkula, H., Paul, D. R. Polymer, 40, p.4237 (1999).

16. Xanthos, M. - "Reactive extrusion: Principles and Practice", Oxforf University Press, New York (1992).

17. Gan,P. P., Paul, D. R. - Polymer 35, p.3513 (1994).

Enviado: $11 / 12 / 03$

Reenviado: 03/03/04

Aprovado: 23/03/04 